\title{
50 Años de la Escuela de Enfermería de Guadalajara
}

\author{
Helena Hernández Martínez ${ }^{1, *}$, Ma Isabel Pascual Benito ${ }^{2}$ \\ 1 Profesora del Departamento de Enfermería y Fisioterapia. Vicedecana - Decana Adjunta de Enfermería de \\ la Facultad de Medicina y Ciencias de la Salud, Universidad de Alcalá. \\ 2 Profesora del Departamento de Enfermería y Fisioterapia, Universidad de Alcalá. \\ * Autora correspondencia: misabel.pascual@uah.es
}

Recibido: 15/05/2019; Aceptado: 27/05/2019; Publicado: 31/05/2019

Resumen: Durante este curso 2018/19, se cumplen 50 años del inicio de los estudios de Enfermería en Guadalajara. En 1968, el Instituto Nacional de Previsión crea la Escuela de Auxiliares Técnicos Sanitarios Femeninos de la Residencia Sanitaria de la Seguridad Social «Fernando Primo de Rivera», vinculada a la Facultad de Medicina de la Universidad de Madrid. En 1980, los estudios pasan a ser universitarios, cambiando la titulación a Diplomatura Universitaria en Enfermería, y el centro a Escuela Universitaria de Enfermería (EUE) INSALUD-Guadalajara adscrita a la Universidad de Alcalá. En 2002, al transferirse las competencias sanitarias pasa a denominarse EUE SESCAMGuadalajara. El 21 de diciembre de 2006 se firma el convenio entre las Consejerías de Sanidad y de Educación y Ciencia de la JCCM y la Universidad de Alcalá por el que se integra la Escuela a la Universidad como centro propio con efecto a partir del curso académico 2006/07, denominándose EUE de Guadalajara y siendo trasladada al Edificio Multidepartamental. Desde los cursos 1980/81 a 2008/09 se diploman 29 promociones. En el curso 2009/10 se implantan los estudios de Grado en Enfermería. En junio de 2011, se constituye la Junta de Centro y en diciembre de ese mismo año, la Escuela pasa a Facultad de Enfermería de Guadalajara. En 2012, como consecuencia del proceso de fusión de Centros, el Grado en Enfermería de Guadalajara pasó a formar parte de la creada Facultad de Medicina y Ciencias de la Salud. Llevando hasta el curso académico actual, 2018/19, tituladas 6 promociones de Graduados/as.

Palabras Clave: Escuela Enfermería Guadalajara; ATS-F Guadalajara; Enfermería Guadalajara.

Abstract: During this 2018/19 academic year, 50 years have passed since the beginning of Nursing studies in Guadalajara. In 1968, the National Institute of Social Welfare created the School of Women's Health Technical Assistants of the Social Security Sanitary Residence "Fernando Primo de Rivera", linked to the Faculty of Medicine of the University of Madrid. In 1980, the studies become university, changing the degree to University Diploma in Nursing, and the center to University School of Nursing (EUE) INSALUD-Guadalajara attached to the University of Alcalá. In 2002, when sanitary competences were transferred, it was renamed EUE SESCAM-Guadalajara. On December 21, 2006, an agreement was signed between the Ministry of Health and Education and Science of the JCCM and the University of Alcalá, through which the School is integrated into the University as its own center with effect from the academic year 2006 / 07, being called EUE de Guadalajara and being transferred to the Multidepartamental building. From the 1980/81 to 2008/09 courses, 29 promotions are awarded. In the 2009/10 academic year, Nursing Degree studies were implemented. In June 2011, it's called Nursing Faculty of Guadalajara. In 2012, as a result of the process of merging Centers, the Degree in Nursing of Guadalajara became part of the created Faculty of Medicine and Health Sciences. Leading up to the current academic year, 2018/19, titled 6 Graduate promotions.resumen en inglés.

Key words: Faculty Nursing Guadalajara; Female Health Technical Assistants; Nursing Guadalajara Spain. 


\section{La Escuela de Ayudantes Técnicos Sanitarios Femeninos}

La creación en 1942 del Seguro Obligatorio de Enfermedad incluye en los artículos 13 y 21 de su Reglamento la prestación del servicio de hospitalización en enfermedad y maternidad, lo que hizo que se creasen nuevos centros sanitarios, entre ellos en Guadalajara, la Residencia Sanitaria «Fernando Primo de Rivera». La existencia en Guadalajara desde 1951 de un moderno centro hospitalario, hizo que su actividad se viera necesitada de un progresivo aumento del número de enfermeras; la población alcarreña estaba estabilizada pero su demanda de servicios sanitarios estaba creciendo; por ello las autoridades sanitarias de Guadalajara pensaron que era el momento de instituir en la nueva Residencia Sanitaria una Escuela de Enfermeras y para ello se movilizaron en los niveles posibles hasta la consecución del objetivo.

La Escuela de Auxiliares Técnicos Sanitarios Femeninos de Guadalajara fue creada en 1968 por el Instituto Nacional de Previsión (INP) con un presupuesto de mantenimiento de 587.300 pesetas. La Junta Rectora de la Escuela fue constituida el 14 de noviembre de 1968, en esa primera reunión, se nombró al profesorado del primer cuatrimestre y se constituyó, así mismo, el tribunal para juzgar el examen de ingreso. El curso se inicia el día 1 de diciembre, aunque estaba pendiente de recibirse la autorización para ello.

El Ministerio de Educación y Ciencia legalizó la Escuela el 21 de enero de 1969. Un escrito del Decano de la Facultad de Medicina de la Universidad Complutense de Madrid decía que «Este ministerio, de conformidad con lo dispuesto en el Decreto de 27 de junio de 1952 y la Orden ministerial de 4 de agosto de 1953, ha resuelto: 1ํㅡㄹ Crear la Escuela de Ayudantes Técnicos Sanitarios Femeninos en Guadalajara de la Residencia Sanitaria de la Seguridad Social «Fernando Primo de Rivera», de dicha localidad, que queda vinculada a la Facultad de Medicina de la Universidad de Madrid». El escrito indica también que la Residencia Sanitaria debía redactar los Estatutos de la Escuela y posteriormente su Reglamento de funcionamiento ajustándose a lo dispuesto en el Decreto de 4 de diciembre de 1953 y la Orden Ministerial de 4 de julio de 1955, en relación al funcionamiento de estas Escuelas; debiendo enviarse ambos documentos al Decanato para que fuesen aprobados.

En la segunda reunión de la Junta Rectora, el 30 de enero de 1969, ésta quedó enterada de que por el Ministerio de Educación y Ciencia había sido aprobada la Escuela, con el encargo de elaborar sus Estatutos y Reglamentos, que fueron aprobados en esa reunión; así mismo en dicha sesión se hicieron los nombramientos de los profesores de las asignaturas del segundo cuatrimestre.

\subsection{Los Estatutos y normativas de la Escuela de ATSF}

Los Estatutos imponían una serie de normas sobre la estructura general de la Escuela, como la composición de la Junta de Gobierno que quedaba formada por el Catedrático Inspector, como Presidente; el Director de la Escuela como Vicepresidente; la Enfermera Jefe de la Escuela y la Secretaria de Estudios, que actuaría como secretaria de la Junta. Dicha Junta ha de reunirse una vez al mes, designará al profesorado, al tribunal encargado de juzgar las pruebas de ingreso, a los tribunales de los exámenes finales y propondrá al INP y a la Universidad Complutense cuantas acciones considere necesarias para mejorar el funcionamiento de la Escuela; se indicaba el personal que dependerá de la Institución, la forma de nombramiento y, por último, se establece el plan pedagógico, que señala que la Escuela tendrá 25 alumnas como máximo y 10 como mínimo en cada curso académico.

Las normas generales de las Escuelas de ATSF establecían que los estudios debían realizarse en régimen de internado, por lo que, en principio las estudiantes tenían su internado en el Colegio de Religiosas Adoratrices, anejo a la Residencia de la Seguridad Social, separado de ella por una valla que fue eliminada para permitir el acceso de las alumnas desde el Colegio a la Residencia donde se desarrollaban las clases teóricas y las prácticas. En abril de 1972 se terminó y se inauguró el Edificio de la Escuela de la calle Ferial, anexo a la Residencia Sanitaria, un edificio funcional y amplio, que, en ese momento, respondía a las necesidades de la enseñanza y el internado, pero pronto además sirvió como válvula de escape para las necesidades asistenciales de la Residencia, que en años siguientes fueron ocupando plantas con camas, consultas, etc. 
La normativa del internado establecía un severo régimen de vida, con horarios estrictos, necesidad de autorizaciones y finalmente se decía que «las alumnas presentarán extremada uniformidad, limpieza e higiene personal, evitando la aplicación de afeites o adornos según las normas que se las den». Indicaba que había que levantarse cada día a las $7.30 \mathrm{~h}$ de la mañana, asistencia a misa voluntaria, puntualidad y uniformidad en todo momento, imposibilidad de ausentarse del internado sin autorización de la Jefe, Secretaria o Instructora de la Escuela y prohibición de las visitas al internado durante la semana, a excepción de los sábados por la tarde y los domingos. Las luces de las habitaciones debían apagarse cada día a las 23.30 como máximo.

Los estudios constarán de tres cursos con enseñanzas teóricas según se señalaba en la Orden Ministerial de 4 de agosto de 1953, complementadas con las enseñanzas de Religión, la Moral Profesional, las Enseñanzas del hogar, la Formación política y la Educación Física, según el Decreto de 28 de noviembre de 1939.

Las enseñanzas prácticas tendrían lugar en todos los servicios de la Residencia Sanitaria «Fernando Primo de Rivera», distribuyéndose las alumnas para el desarrollo de las prácticas en grupos de cinco como máximo y repartidas por la Institución en turnos rotativos, con una duración de 4 horas diarias durante el primer curso y de 6 horas diarias en $2^{\circ}$ y $3^{\circ}$ cursos, realizando prácticas en servicios de urgencia nocturnos y días festivos. La Facultad de Medicina fijaba el calendario escolar de la Escuela e indicaba que «con el fin de lograr una mejor formación práctica se reducirán las festividades a las de máxima festividad religiosa, tanto en épocas de Navidad, Semana Santa y otras festividades. Semanalmente se facilitarán períodos de descanso los sábados por la tarde y domingo con arreglo a turnos rotativos y las vacaciones de verano serán de 45 días, tomadas en dos turnos».

La asistencia a prácticas era obligatoria entre las 9 y las 13 horas, el «Ofrecimiento de Obras» en la capilla de la Residencia, extremada uniformidad con el pelo recogido bajo la toca, riguroso respeto hacia jefes de clínica, médicos, residentes, etc. y no administración de ningún medicamento sin prescripción médica o permiso de la enfermera de turno, quedaba prohibido fumar en horas de servicio, así como la obligatoriedad de acudir al «estudio» de 20 a 21 horas en la Sala de Juntas. Al ingresar, las alumnas recibían indicaciones de cómo debía ser el uniforme; este se componía de bata, manguitos, delantal, gorro, medias y zapatos. La bata sería de tela de tergal, abierta por delante de arriba abajo, con botones de pasta blancos; la falda tendría un vuelo de 2 metros. Respecto al gorro, debía ser de organdí suizo blanco, de $41 \mathrm{~cm}$ de ancho, 18 de altura en el centro y en los extremos de $28 \mathrm{~cm}$, haciendo dos pinzas en cada lado, debiendo doblar la vuelta. Las medias eran de color gris perla y los zapatos negros.

Se estableció una Asesoría Eclesiástica para la Escuela, especificando el Reglamento que el «profesor de Religión y Moral Profesional será nombrado por la Autoridad Eclesiástica de la Provincia y tendrá entre sus funciones específicas de Profesor de las citadas disciplinas la asesoría religiosa de la Escuela».

Para acceder como estudiante a la Escuela, los Estatutos establecen cómo han de hacerse los exámenes de ingreso, que consistían en un examen médico, una entrevista personal con la alumna para analizar con ella sus antecedentes personales, escolares y motivacionales; un examen psicotécnico basado en test que valorarían el «perfil profesiográfico de la carrera de enfermera» y finalmente un examen de ingreso basado en el programa aprobado por el ministerio de Educación y Ciencia. Otros requisitos que debían reunir las aspirantes para ser admitidas como alumnas, era tener 17 años cumplidos, ser presentada «por dos personas de solvencia moral reconocida» y añadir una carta de su puño y letra en la que razonase el por qué deseaba seguir los estudios de enfermera.

El Reglamento indicaba la obligatoriedad de matricularse una vez aprobado el examen de ingreso y que no sería admitida ninguna alumna que hubiese sido expulsada de otra Escuela; así como la potestad de la Escuela para seleccionar durante el primer trimestre a las alumnas y sólo permitir continuar sus estudios a aquellas que hayan demostrado poseer condiciones físicas, morales, intelectuales y vocación suficiente para el ejercicio de la profesión.

En octubre de 1968 se convocó públicamente el primer examen de ingreso para acceder a las 15 plazas convocadas. 
El segundo año, de las 47 aspirantes sólo ingresaron 14 y se decidió no realizar convocatoria de nuevo ingreso el año siguiente pues con las alumnas de $1^{\underline{0}}$ y $2^{\underline{0}}$ se cubría la capacidad de acogida del área residencial del Colegio de Adoratrices. Este fue un motivo para acelerar los trámites de construcción del nuevo edificio para la Escuela en la C/ Ferial.

En 1978 con la creación de la Universidad de Alcalá de Henares, los estudios sitos en Guadalajara, Magisterio y Enfermería, que hasta ahora dependían de la Universidad Complutense pasan a depender de la Universidad de Alcalá de Henares. Esto supuso en la Escuela de ATSF, la constitución de un nuevo Órgano de Gobierno, que quedó formado por un Catedrático Inspector de la Universidad de Alcalá, Director del Hospital, Enfermera Jefe de la Escuela, Jefa de Estudios de la Escuela, Jefa de Enfermería del Hospital, Administrador y dos vocales.

Los estudios de ATSF se prolongaron hasta 1978, titulándose 10 promociones de quince alumnas cada una.

\section{La Escuela Universitaria de Enfermería de Guadalajara}

En el BOE de 22 de agosto de 1977 se publicaba el R.D. 2128/77 de 23 de julio que proponía la «Integración en la Universidad de las Escuelas de ATS como Escuelas Universitarias de Enfermería (EUE)»; de acuerdo a esto, el 3 de septiembre de 1977, se solicitaba la autorización para la conversión de la Escuela de ATSF en EUE, a lo que se sumaba la firma, el 11 de abril de 1978, del concierto entre la Universidad de Alcalá de Henares y el INP para la utilización de la Residencia Sanitaria como Hospital docente, colaborador único de la Facultad de Medicina de dicha Universidad.

En julio de 1978 el INP notificó a la Escuela la no autorización para su transformación en EUE, indicando que no se convocasen plazas para el curso 1978-1979, continuándose con los cursos $2^{2}$ y y $3^{\circ}$ hasta su extinción. Pero el personal directivo y docente de la Escuela redoblaron su esfuerzo por conseguir la transformación en Escuela Universitaria de Enfermería y el 27 de julio de ese año se envió al Rector de la Universidad de Alcalá de Henares la solicitud de autorización para la reconversión de la Escuela.

Tras su valoración en la Junta de Rectores, en base a la Orden del Ministerio de Educación y Ciencia de 20 de octubre de 1978, por la que se establecen las condiciones específicas a cumplir por las Escuelas Universitarias de Enfermería en lo referente, entre otros, la estructura física, el límite máximo y mínimo de alumnos por curso y el desarrollo de actividades docentes en Hospitales con acreditación docente; se informa favorablemente el 11 de mayo y por fin, el 26 de septiembre de 1980, el R.D. 2235/80 daba por hecha la conversión en Escuela Universitaria de Enfermería adscrita a la Universidad de Alcalá.

La Universidad de Alcalá de Henares nombró un Delegado de Evaluaciones, quedando integrado el órgano de gobierno, llamado Junta de Patronato, por el Director Provincial del Instituto Nacional de la Salud (INSALUD) de Guadalajara; el Delegado de la Universidad; el Director de la Escuela y los representantes del profesorado.

Así, en 1980 se inician en Guadalajara los Estudios Universitarios de Enfermería, cambiando la titulación a Diplomados/as Universitarios/as en Enfermería, y el centro a Escuela Universitaria de Enfermería del INSALUD-Guadalajara adscrita a la Universidad de Alcalá. La Escuela siguió funcionando como lo había hecho anteriormente como Escuela de ATS-F en cuanto al profesorado que impartía la docencia, médico en su gran mayoría, y las personas dedicadas a su gestión diaria Enfermera Jefe y monitoras de la misma. Pero, supuso un gran cambio no tanto por el número de admitidos, si no porque podían incorporarse a los estudios por primera vez en la Escuela estudiantes masculinos.

Las plazas de nuevo ingreso autorizadas por la Universidad eran de 40 desde la primera promoción de 1980-1983, esta promoción para acceder como estudiante tuvo que realizar un examen de ingreso que se realizó en dependencias de la Universidad de Alcalá consistente en preguntas tipo test con nivel de BUP puesto que ese primer año no se exigió la selectividad para el ingreso.

La formación teórica se realizaba en el Edificio de la Escuela de 16 horas a 20 horas, todos los días de la semana y en las asignaturas del currículum ya no existen las asignaturas de hogar, moral, etc típicas de los estudios de ATS-F. 
Las prácticas se realizaban de lunes a viernes y de 8 a 15 horas en el nuevo Hospital General y Universitario del INSALUD de Guadalajara, inaugurado en 1981. La primera promoción, en su primer curso inicio dichas prácticas en la Residencia Sanitaria y colaboró en el traslado de enfermos al nuevo Hospital, donde termino de realizar las prácticas de $1^{1} \mathrm{y}$ las del resto de cursos. El uniforme de los estudiantes era tipo pijama, con la parte superior abotonada en el lateral izquierdo y la parte inferior pantalón o falda a elegir para las chicas, la inmensa mayoría, por no decir la totalidad eligió pantalón. El color del uniforme era azulón, por lo que pronto fue denominado el estudiantado en prácticas "pitufos".

Tras la Ley de Reforma Universitaria (1983) que habilita a los diplomados universitarios para formar parte del cuerpo docente universitario, supuso la transformación del puesto de Enfermera Jefe en el de Directora Técnica. Esto produjo el cambio estructural de la situación del claustro. Hasta entonces perduró la estructura de las Escuelas de ATSF donde sólo existían tres profesoras enfermeras a dedicación completa siendo el resto del profesorado médico a dedicación parcial. Se amplían las plazas de profesorado a dedicación completa a diez que se ocupan todas ellas por enfermeras, potenciándose la impartición de la docencia por enfermeras, llegando al punto en que en 1992 había más profesoras enfermeras que profesores médicos.

Posteriormente, en 1988 se cambia por parte del Rector la denominación de Directora Técnica a Directora de Escuela pasando a corresponderla la Presidencia de la Junta de Patronato de la Escuela, quedando como vocales el Delegado de la Universidad de Alcalá, el Director Provincial del INSALUD de Guadalajara, el Gerente del Hospital del INSALUD de Guadalajara, la Dirección de Enfermería de Atención Especializada, la Dirección de Enfermería de Atención Primaria, dos representantes del profesorado de la Escuela con dedicación completa, un representante del profesorado a dedicación parcial, los alumnos delegados de cada uno de los tres cursos y la Secretaria de la Escuela, que actúa como secretaria de la Junta.

También en el curso académico de 1988-1989 se cambia el color del uniforme de los estudiantes de azulón a blanco.

En 2000, 25 de abril, el INSALUD aprueba el nuevo Reglamento de Régimen Interior de las Escuelas Universitarias de Enfermería dependientes del Instituto Nacional de la Salud, donde se reglamentan los órganos de gobierno y dirección como la Junta de Patronato, el Claustro, la Dirección y Secretaría de la Escuela; la comunidad escolar con la reglamentación del profesorado y los derechos y deberes de los alumnos; de la organización de las enseñanza y la gestión económica y presupuestaria de la Escuela. A partir de entonces, la presidencia de la Junta de Patronato pasa ser ocupada por la persona que ostenta el cargo de Director Gerente del Hospital General Universitario de Guadalajara, la Directora de la Escuela ocupa una vocalía.

Durante el curso académico 2001/02 se crea y habilita espacio para la Delegación de Alumnos, se codifican en el Sistema Internacional de Bibliotecas los libros existentes en la Biblioteca por parte de una becaria del Servicio de Bibliotecas de la Universidad de Alcalá, se completa la plantilla de profesorado a dedicación completa a 10, se instaura para el acceso a la figura de profesor a dedicación completa y profesor a dedicación parcial el baremo utilizado para tal fin en la Universidad de Alcalá, se incorpora el turno de tarde, de 15 a 22 horas para el desarrollo de las prácticas clínicas de los estudiantes en el HU de Guadalajara y los Centros de Atención Primaria del Área de Guadalajara y se potencian las gestiones para la integración de la Escuela en la Universidad de Alcalá.

El 1 de enero de 2002, la Junta de Comunidades de Castilla-La Mancha asume las competencias en materia de salud, creándose el Servicio de Salud de Castilla-La Mancha (SESCAM). La Escuela es transferida con el resto de competencias sanitarias por lo que pasa a ser de dicho sistema de salud y a denominarse Escuela Universitaria de Enfermería-SESCAM de Guadalajara.

Para el funcionamiento de la Escuela sigue en vigor el Reglamento de Régimen Interior de las Escuelas Universitarias de Enfermería dependientes del Instituto Nacional de la Salud del 2000, manteniéndose como órganos de gobierno y representación en la Escuela, la Junta de Patronato, La Dirección de la Escuela, el Claustro de profesores y la Delegación de Alumnos. 


\subsection{Integración de la Escuela de Enfermería de Guadalajara en la Universidad de Alcalá}

En 1999 se inicia el proceso de convergencia hacia un Espacio Europeo de Educación Superior (EEES) con la Declaración de Bolonia (1999). La legislación española se adecua con los RD. 1125/2003 en el que se establece el Sistema Europeo de Transferencia de Créditos ECTS en las titulaciones universitarias de carácter oficial y validez en todo el territorio nacional, el RD 55/2005 de 21 de enero la por el que se establece la estructura de las enseñanzas universitarias y se regulan los estudios universitarios oficiales de Grado y la necesidad de que una vez elaborados los planes de estudios deberán ser verificados por el Consejo de Universidades, ateniéndose a las normas establecidas por la Agencia Nacional de Evaluación de la Calidad y Acreditación (ANECA), para ello los centros deberán elaborar una memoria de verificación.

Ante esta situación, es necesaria la adecuación de los estudios de Diplomado en Enfermería de la Escuela de Enfermería-SESCAM de Guadalajara en estudios de Grado en Enfermería, pero además necesita que los recursos materiales y personales que deben figurar en la memoria de verificación sean aprobados por ANECA y la situación de la Escuela no es la adecuada. Los recursos que ofrece el Edificio de la Escuela de la Calle Ferial, otrora funcional y amplio, ya no proporcionaba los recursos necesarios para la enseñanza de Grado. Por todo esto, se inician conversaciones con el Consejero de Sanidad y el Gerente del SESCAM por un lado y la Universidad de Alcalá, por otro, para darle una solución ante la posibilidad del cierre de la Escuela. La solución aceptada por todos es su integración en la Universidad de Alcalá.

El 21 de diciembre de 2006, en Guadalajara, se firma el Convenio entre la Consejería de Sanidad de la Junta de Comunidades de Castilla-La Mancha, representada por el Consejero de Sanidad D. Roberto Sabrido Bermúdez, la Consejería de Educación y Ciencia de la Junta de Comunidades de Castilla-La Mancha, representada por el Consejero de Educación y Ciencia D. José Valverde Serrano y por la Universidad de Alcalá, representada por su Rector D. Virgilio Zapatero Gómez, por el que se integra la Escuela Universitaria de Enfermería-SESCAM de Guadalajara a la Universidad de Alcalá con efecto a partir del curso académico 2006/07.

Desde ese momento la Escuela se convierte en un centro más de la Universidad de Alcalá, pasa a denominarse Escuela Universitaria de Enfermería de Guadalajara, como órgano de gobierno se crea una Comisión Gestora y es trasladada al Edificio Multidepartamental que la Universidad de Alcalá tiene en el Campus de Guadalajara en el mes de septiembre, iniciándose ya el curso 2007/08 en la nueva ubicación. Ofertándose 75 plazas de nuevo ingreso y se realizan para las prácticas clínicas del estudiantado convenios con distintas instituciones y centros sociosanitarios que garantizan la calidad de la enseñanza. El profesorado a dedicación completa se integra en la Universidad como Titulares de Escuela Universitaria en Comisión de Servicio, pasando a formar parte del Departamento de Enfermería y se convocan 25 plazas de Profesor Asociado en Ciencias de la Salud para el Área de Salud de Guadalajara. Desde el curso académico 1980/81 hasta el curso académico 2008/09 se titulan 29 promociones de Diplomados en Enfermería, se diploman aproximadamente 1300 estudiantes.

\section{Grado en Enfermería}

Las condiciones que debe cumplir el título de Grado en Enfermería que habilite para el ejercicio de la profesión regulada de Enfermera se recogen en la 3648 Resolución de 14 de febrero de 2008, de la Secretaría de Estado de Universidades e Investigación, por la que se da publicidad al Acuerdo de Consejo de Ministros de 8 de febrero de 2008, por el que se establecen las condiciones a las que deberán adecuarse los planes de estudios en virtud de lo dispuesto en los artículos 2 y 7 de la Ley 44/2003, de 21 de noviembre, de ordenación de las profesiones sanitarias, en la que se contienen los aspectos básicos de las profesiones y la Directiva 2005/36/CE del Parlamento Europeo y del Consejo.

A su vez, la Orden CIN/2134/2008, de 3 de julio, establece los requisitos para la verificación por el Consejo de Universidades de los títulos universitarios oficiales de Grado en Enfermería. Dentro de estos requisitos define las 18 competencias específicas de titulación que los estudiantes deben adquirir, establece la duración de 240 créditos europeos, los tres módulos que debe incluir como mínimo, Módulo de Formación Básica de 60 ECTS, Módulo de Ciencias de la Enfermería de 60 ECTS 
y Módulo de Prácticas Tuteladas y Trabajo Fin de Grado de 90 ECTS, y las competencias que debe adquirir el estudiante en cada uno de los módulos.

Teniendo en cuenta la legislación y normativas la EU de Enfermería de Guadalajara y la EU de Enfermería y Fisioterapia, ambas de la Universidad de Alcalá, constituyen una Comisión Mixta para elaborar la memoria para la verificación de Grado en Enfermería de la Universidad de Alcalá. El 13/05/2009 se termina de elaborar y se envía la Memoria para la solicitud de verificación del Título de Grado en Enfermería. La Consejería de Educación en BOCM de 21 de agosto de 2009 autoriza a la Universidad de Alcalá a la implantación en el curso 2009-2010 del Grado en Enfermería de Guadalajara. Publicándose posteriormente en el BOE de 9 de octubre de 2009 el carácter oficial del Título y su inscripción en el Registro de Universidades, Centros y Títulos. El Plan de estudios conducente al título de Graduado en Enfermería por la Universidad de Alcalá es publicado el BOE de 23 de marzo de 2010.

En el curso académico 2009/10 se implantan los estudios de Grado en Enfermería por la Universidad de Alcalá en la Escuela de Enfermería de Guadalajara.

La nueva titulación de Grado, rompe el techo de cristal de la Diplomatura, en cuanto al desarrollo de la investigación y el desarrollo académico para los egresados. Los nuevos graduados pueden acceder a los estudios de Máster y Doctorado, no siendo ya necesario para este desarrollo el rodeo dado por numerosos Diplomados en Enfermería estudiando una licenciatura que les habilitará para dicho acceso.

En junio de 2011 se constituye la Junta de Centro de la Escuela de Enfermería de Guadalajara, disolviéndose la Comisión Gestora. En diciembre de ese mismo año la Escuela de Enfermería de Guadalajara pasa a denominarse Facultad de Enfermería de Guadalajara.

En 2012, como consecuencia del proceso de fusión de Centros, llevado a cabo por el equipo rectoral de la Universidad de Alcalá, el Grado en Enfermería de Guadalajara pasó a formar parte de la creada Facultad de Medicina y Ciencias de la Salud junto con los grados de CCAFYDE, Enfermería, Fisioterapia y Medicina.

En marzo de 2016 se inicia el proceso de elaboración del autoinforme para la Renovación de la Acreditación, terminándose en septiembre del mismo año. Una vez tramitado a la Fundación para el Conocimiento Madrid I+D y tras la evaluación externa, los Estudios de Enfermería de Guadalajara reciben el 12 de julio de 2017 el Informe Final de Renovación de la Acreditación Favorable.

Desde la implantación del Grado en Enfermería de Guadalajara por la Universidad de Alcalá, curso 2009/10, hasta el curso académico actual, 2018/19, llevan tituladas 6 promociones de Graduados/as en Enfermería.

Desde su creación la Escuela de Enfermería de Guadalajara ha sido asumida por los/as ciudadanos/as de la ciudad como una institución propia, formando parte del desarrollo su social. Esta imbricación social puede observarse en la cantidad de artículos periodísticos en los que ha estado la Escuela desde su creación hace 50 Años. Las fiestas de Graduación eran recogidas en la prensa y las Fiestas del Patrón de la Escuela que se celebraban en el parking del Edifico del Ferial reunía la juventud de Guadalajara.

Los egresados de cualquiera de las titulaciones que han tenido lugar a lo largo de la historia de la Escuela de Enfermería de Guadalajara, ATS-F, Diplomado en Enfermería y Graduado en Enfermería, han demostrado su gran profesionalidad en la actividad asistencial desarrollada, en los puestos de gestión asistencial, en la docencia, en los puestos de gestión académica, y, en general, en todo el ámbito sociosanitario. Han sido egresados requeridos en hospitales del Área de Guadalajara y del Área de Madrid, profesionales comprometidos socialmente, ocupando puestos de responsabilidad en Asociaciones, ONGs, sindicatos y la esfera política. Y, desde la oferta de las plazas de EIR para las Especialidades de Enfermería han ocupado los primeros puestos.

\section{Celebración del 50 Aniversario de la creación de la Escuela de Enfermería de Guadalajara}

Por toda la historia anterior reseñada y como no podía ser de otra manera, en este curso académico 2018-2019 se ha celebrado el 50 Aniversario de la actividad docente de nuestro Centro. Para ello, la celebración se ofrece como un espacio y un tiempo de encuentro abierto para aquellas 
personas interesadas en la historia de la Escuela y la formación enfermera tan ligadas en el tiempo. Por esto, la estructura y planificación de las actividades del 50 Aniversario desarrolladas a lo largo del curso académico 2018-2019, se han basado en:

- Una exposición histórica con material docente teórico, documentos, fotos y material práctico y asistencial que puede ser visitada en el Hall del Edificio Multidepartamental.

- Un ciclo de mesas redondas, el último miércoles de cada mes, terminando en marzo, cada una de ellas dedicada a una de las etapas de la historia de la Escuela (ATS-F, Diplomatura y Grado) y una mesa final dedicada al presente y futuro de la Enfermería.

- Un concurso de fotografía y un concurso de relatos cortos con el tema central de Enfermería. En el acto académico de clausura de las actividades del 50 Aniversario coincidente con el patrón de los Estudios, el Día Internacional de Enfermería, el día 10 de mayo, se hizo también entregada de los premios.

No quisiéramos acabar este recorrido por la historia de la Escuela de Enfermería de Guadalajara sin dar las gracias a las autoridades de las distintas instituciones que han apoyado a la Escuela en los momentos decisivos de su historia, a todo el profesorado que ha participado en la docencia en estos 50 años, a todo el personal de administración y servicios que han trabajado en y para la Escuela, a las enfermeras y enfermeros de la instituciones sociosaniatrias por su colaboración en el desarrollo de la practicas clínicas, al estudiantado que ha pasado por sus aulas y, en general a la sociedad guadalajareña donde la Escuela ha crecido incardinada. A todos/as igracias! por la calidad, prestigio y utilidad social que la Escuela mantiene desde sus orígenes.

Conflictos de Intereses: los autores no declaran conflicto de intereses.

\section{Referencias Bibliográficas}

1. VVAA. Escuela Universitaria de Enfermería. 30 años de la Escuela Universitaria de Enfermería INSALUD de Guadalajara. AACHE, Guadalajara, 1999.

2. Helena Hernández Martínez, Mํa Isabel Pascual Benito (Coordinadoras) Cuarenta años de la Escuela Universitaria de Enfermería. Servicio de Publicaciones Universidad de Alcalá. 2009.

(C) 2019 por los autores; Esta obra está sujeta a la licencia de Reconocimiento 4.0

Internacional de Creative Commons. Para ver una copia de esta licencia, visite http://creativecommons.org/licenses/by-nc-nd/4.0/. 